\title{
HIGHER ORDER MODULATION INTERSYMBOL INTERFERENCE CAUSED BY TRAVELING-WAVE TUBE AMPLIFIERS
}

\author{
Carol L. Kory ${ }^{1}$ and Monty Andro ${ }^{2}$ \\ 'Analex Corporation ${ }^{2}$ NASA Glenn Research Center ${ }^{*}$ \\ 21000 Brookpark Road, MS 54-5 \\ Cleveland, $\mathrm{OH} 44135$ \\ Carol.L.Kory@grc.nasa.gov
}

\begin{abstract}
For the first time, a time-dependent, physics-based computational model has been used to provide a direct description of the effects of the traveling wave tube amplifier (TWTA) on modulated digital signals. The TWT model comprehensively takes into account the effects of frequency dependent AM/AM and AM/PM conversion; gain and phase ripple; drive-induced oscillations; harmonic generation; intermodulation products; and backward waves. Thus, signal integrity can be investigated in the presence of these sources of potential distortion as a function of the physical geometry and operating characteristics of the high power amplifier and the operational digital signal. This method promises superior predictive fidelity compared to methods using TWT models based on sweptamplitude and/or swept-frequency data.

First, the TWT model using the three dimensional (3D) electromagnetic code MAFIA is presented. Then, this comprehensive model is used to investigate approximations made in conventional TWT black-box models used in communication system level simulations. To quantitatively demonstrate the effects these approximations have on digital signal performance predictions, including intersymbol interference (ISI), the MAFIA results are compared to the system level analysis tool, Signal Processing Workstation (SPW), using high order modulation schemes including 16 and 64-QAM.
\end{abstract}

\section{INTRODUCTION}

One of the bottlenecks in achieving high data rate throughput for satellite communications is the distortion caused by the traveling-wave tube amplifier (TWTA) used in the communication system high power transmitter. There are two major types of distortion caused by the TWTA: adjacent channel interference (ACI), which is a result of the nonlinearity of the device, and intersymbol interference (ISI), which is suspected to be caused by TWT dispersion resulting in gain variation with frequency, frequency-dependent reflections within the TWT resulting in gain/phase ripple.

$\mathrm{ACl}$ is a well-researched area and much effort has been put into its mitigation using linearizers and predistortion techniques. ISI, on the other hand, is a fairly new concept that has garnered significant attention since communications incorporating large system bandwidths and high order modulation schemes required for very high data rates are particularly sensitive to ISI. The effect of the TWT on ISI is not well understood, and thus effective mitigation methods are not well established. To experimentally investigate the correlation between ISI and the TWT would be prohibitively expensive, as it would require building numerous TWTs as well as the digital hardware, therefore making accurate modeling tools critical.

To predict if adequate signal detection will be achieved in the presence of the TWT, standard practice involves using communication system level software. These models use a nonlinear, black box model to represent the TWT. Currently, SPW ${ }^{1}$ is used in-house at NASA Glenn Research Center (GRC), where the behavior of the TWT is characterized by a memoryless envelope model. The model parameters are obtained from a lookup table with the AM/AM (output power versus input power) and AM/PM (output phase versus input power) conversions, which are typically obtained by sweeping the input amplitude at the center frequency either experimentally or by using conventional frequency domain TWT codes such as TWA $3^{2}$. Thus,

\footnotetext{
"Work supported by NASA's Computation Information and Communications Technology program in Code R

${ }^{\circledR}$ Copyright 2002 The American Institute of Aeronautics and Astronautics Inc. All rights reserved.
} 
the TWT characteristics are assumed constant (memoryless) over the bandwidth of the simulated signal. In addition, the model does not account for the memory effects of signal reflections and gain/phase ripple. As data rate requirements increase and complex, wide-band, digital signals are employed, the assumptions made in conventional system level modeling become less accurate.

In an effort to improve the accuracy of the TWTA model used in communication system level modeling, a fully 3D, time-dependent, TWT interaction model has been developed ${ }^{3}$ using the electromagnetic particle-incell (PIC) code MAFIA (Solution of MAxwell's

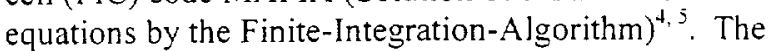
TWT model comprehensively takes into account the effects of frequency dependent AM/AM and AM/PM conversion; gain and phase ripple due to reflections, drive-induced oscillations; harmonic generation; intermodulation products; and backward waves. This physics based TWT model can be used to give a direct description of the effects of the nonlinear TWT on the operational signal as a function of the physical device. The actual geometry of the device is taken into account allowing standard or novel TWT's to be investigated. The user is also able to define arbitrary excitation functions so that higher order modulated digital signals can be used as input and computational correlation of ISI with TWT parameters can be directly conducted. This type of system level nonlinear model can be expected to possess superior predictive fidelity compared to models based on swept-tone, sweptamplitude data. The latter data is partial because superposition does not apply in the nonlinear device, and the interactions between frequency components in a realistic signal are not captured ${ }^{6}$.

The MAFIA TWT interaction model will be described, and results will be presented where this model was used to investigate the accuracy of several assumptions made in TWT black-box models. In addition, digital signal performance, including ISI, is compared using direct data input into the MAFIA model and using the system level analysis tool, SPW, for several high order modulation schemes.

The MAFIA model is computationally intensive (CPU times of several hours), thus it cannot replace conventional nonlinear black-box models for system level simulations. The MAFIA time-dependent data can be used, however, in place of experimental data as input to develop system level, nonlinear, black box models for the TWT with memory such as in ${ }^{7}$ where experimental data is used to obtain model parameters.

\section{MAFIA TWT MODEL}

The TWT used as a model for this study is a Northrop Grumman, 2-6 GHz TWT producing 20 - $30 \mathrm{~dB}$ of gain over the bandwidth. It is presently located at the University of Wisconsin, and designated the XWING (eXperimental WIsconsin Northrop Grumman).

The MAFIA TWT model is a fully 3D representation of the TWT slow-wave circuit, input/output coupling, electron beam and magnetic focusing. The model includes a rectangular tape, helical, slow-wave circuit. The simulated input/output coupling, which couples the RF signal in and out of the circuit, includes a coax with extended center pin to touch the helical circuit. The Sparameters were calculated using MAFIA as described $\mathrm{in}^{3}$, and the return loss is plotted as a function of frequency for the final coupler design in Figure 1 . The match is excellent around $4.2 \mathrm{GHz}$ with a return loss of $-35 \mathrm{~dB}$. Therefore, we choose this value as our center frequency $f_{c}$ for all simulations.

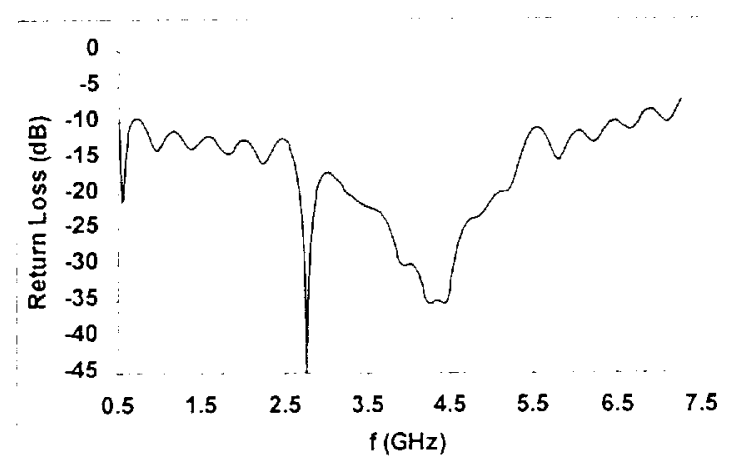

Figure 1 Return loss for simulated XWING coupler design

To complete the helical interaction model, a $2.8 \mathrm{kV}$, 0.22 A electron beam was defined consistent with the XWING operating parameters, and a uniform focusing field of 0.15 Tesla was implemented. Conductor and dielectric losses were assumed zero in all simulations Because of the computational intensity added by modeling in 3D, the model was limited to a small signal gain of $12.5 \mathrm{~dB}$ at $4.2 \mathrm{GHz}$, which is sufficient for this study.

To validate the MAFIA model, results were compared to the 2.5D, multi-signal, frequency domain, TWT code, TWA $3^{2}$, which has compared well with experimental data in the past. The power transfer characteristics at $4.2 \mathrm{GHz}$ were obtained using both MAFIA and TWA3. The output power and phase shift from small-signal (i.e. AM/AM and AM/PM) as a function of input drive power are shown in Figure 2 The agreement in output power is very good. 
The phase plot shows a phase change $\Delta \phi$ between an input power of $30 \mathrm{~dB}$ below saturation and saturation of 10.3 and 18.9 degrees for the TWA3 and MAFIA models, respectively. The phase is very sensitive to the beam behavior and its interaction with the RF signal. Since significant differences exist in the manner in which each code represents the electron beam, this agreement is considered quite good.

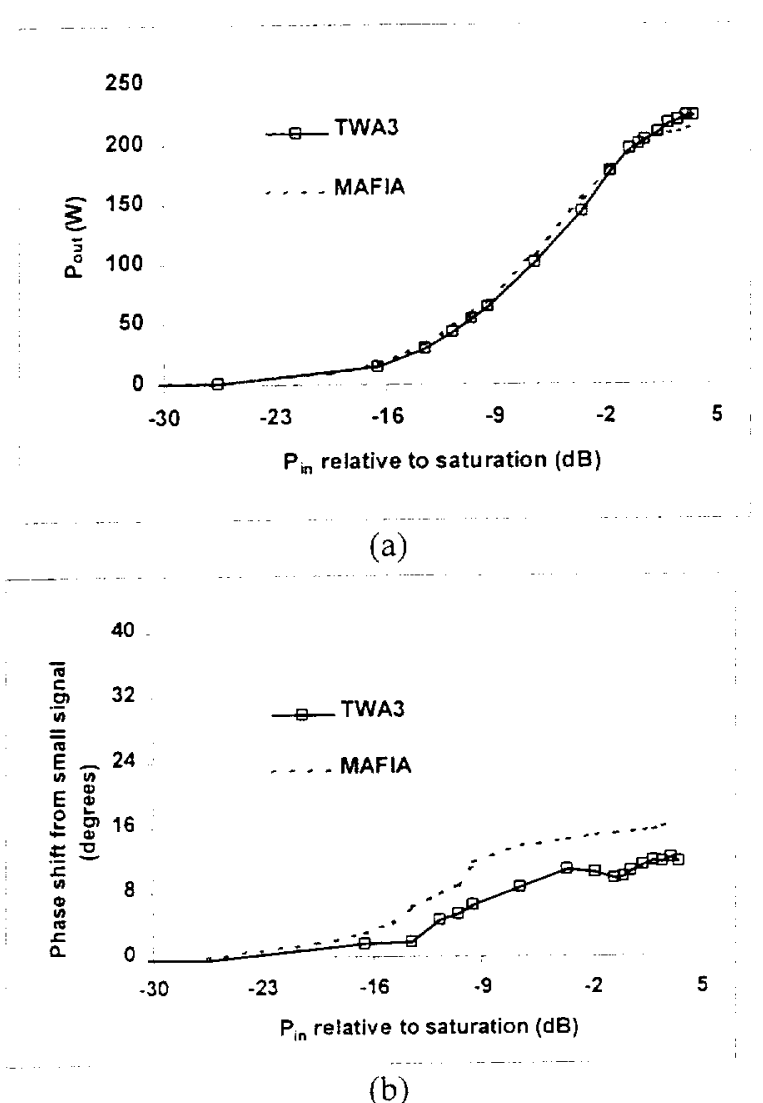

Figure 2 Simulated (a) output power and (b) phase as a function of input power at $4.2 \mathrm{GHz}$

\section{INVESTIGATION OF SYSTEM LEVEL TWT MODEL ASSUMPTIONS}

Conventional, system level, nonlinear, black box models representing the TWT vary in complexity ${ }^{6}$, but most make several assumptions regarding the operation of the high power amplifier. In particular, it is common to neglect the following TWT operational characteristics:

1. Dependence of $\mathrm{AM} / \mathrm{AM}$ and $\mathrm{AM} / \mathrm{PM}$ with frequency

2. Effects of reflections and second harmonics

3. Differences between broadband and single-tone excitations

4. Dependence of gain and phase ripples with drive power

5. Dependence of output spectrum with operational signal

The TWT box model used in SPW does not account for any of these characteristics. The significance of neglecting them is investigated quantitatively in this section using the MAFIA model.

\section{Dependence of AM/AM and AM/PM with frequency}

To demonstrate the variation of gain and phase with frequency for the XWING TWT model, the power transfer characteristics were calculated using MAFIA at several frequency points using single-tone excitation. The gains are plotted in Figure 3 versus input power relative to the saturation point calculated at $4.2 \mathrm{GHz}$. From here forth, $\mathrm{P}_{\text {in }}$ will be specified relative to saturation at $4.2 \mathrm{GHz}$. Compared to $4.2 \mathrm{GHz}$, the gain at $3.2 \mathrm{GHz}$ shows a reduction in small-signal gain of $1.1 \mathrm{~dB}$. At $5.2 \mathrm{GHz}$ and $5.5 \mathrm{GHz}$, there is a smallsignal reduction of $0.3 \mathrm{~dB}$ and $0.5 \mathrm{~dB}$, respectively. The gain calculations have not been calibrated to take the variation of return loss with frequency into consideration. Consequently, the deviation in gain from the $4.2 \mathrm{GHz}$ is a result of the TWT dispersion and coupler match, as would be representative of an experimental TWT. There is a deviation in $\Delta \phi$ by approximately five degrees between the $3.2 \mathrm{GHz}$ and $5.5 \mathrm{GHz}$ cases.

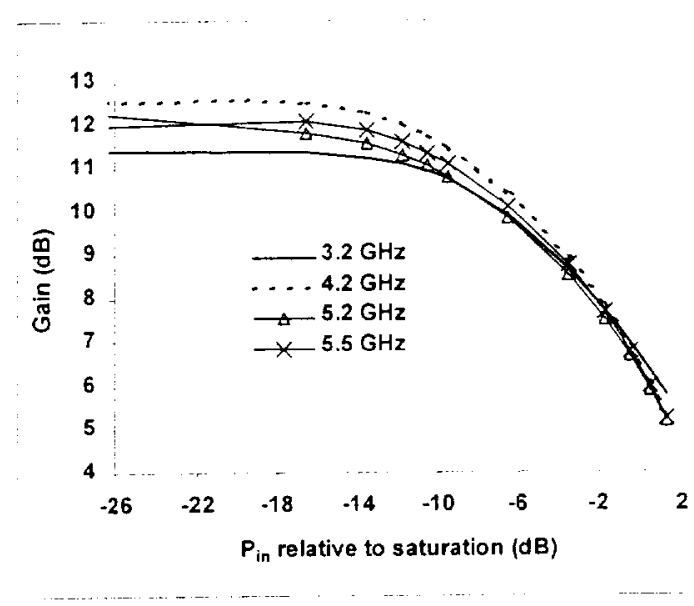

Figure 3 MAFIA gain for several frequency points

\section{Effects of reflections and second harmonics} Several single-tone excitations were simulated with MAFIA and the TWT output was recorded at the first arrival of the RF signal at the output port (without reflections), and at a point in time after the signal had time to make one feedback path around the TWT and 
return to the output port (with reflections). An operating point of $5 \mathrm{~dB}$ input backoff (IBO) relative to saturation was chosen as might be used for high data rate communications using high-level modulation schemes.

Comparisons of the gain and strength of the second harmonic frequency $f_{2}$ relative to the fundamental frequency $f_{1}$ with and without reflections are shown in Figure 4 . The average absolute difference in gain between the two cases is $0.28 \mathrm{~dB}$ across the bandwidth. Interestingly, the gain plot for the case with reflections shows a peak-to-peak variation of as much as $0.72 \mathrm{~dB}$ around $4.2 \mathrm{GHz}$. However, conventional theory predicts negligible ripple where there are negligible reflections ${ }^{8}$, and from the return loss of the circuit plotted in Figure 1, we know that the match at this frequency is $-35 \mathrm{~dB}$. For this circuit the ripple at 4.2 $\mathrm{GHz}$ can be attributed to the second harmonic ( 8.4 $\mathrm{GHz}$ ) interaction with the beam and its large reflections at the input/output couplers. The second harmonic is present when the signal hits the output the first time due to the nonlinearity of the TWT; its strength is dependent on the operating point of the TWT, and thus the level of nonlinearity. When the second harmonic component of the signal hits the output, a large amount is reflected back into the TWT at the output port. By extrapolating the data in Figure 1, we might assume that the return loss at $8.4 \mathrm{GHz}$ will be about $-5 \mathrm{~dB}$.

Therefore, a large portion of the signal will be reflected back, and again a large portion will be reflected when the signal hits the input port. Since synchronism still holds, the second harmonic will grow as it again travels in the forward direction. The electrical length, or manner in which the phase adds or cancels as a function of frequency, determines the behavior of the ripple. In turn, the fundamental gain exhibits a similar ripple that is shifted by 180 degrees. For example, notice from Figure 4 that when the relative second harmonic strength hits a maximum, the gain of the fundamental hits a minimum and vice versa. This phenomena cannot be predicted by conventional frequency domain codes.

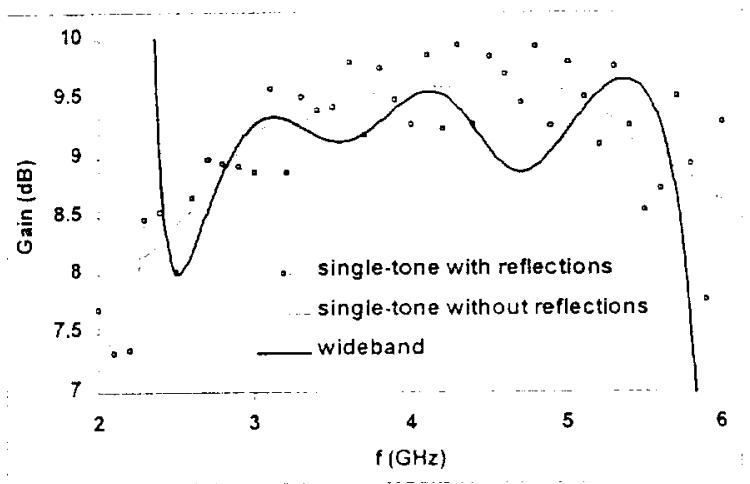

(a)

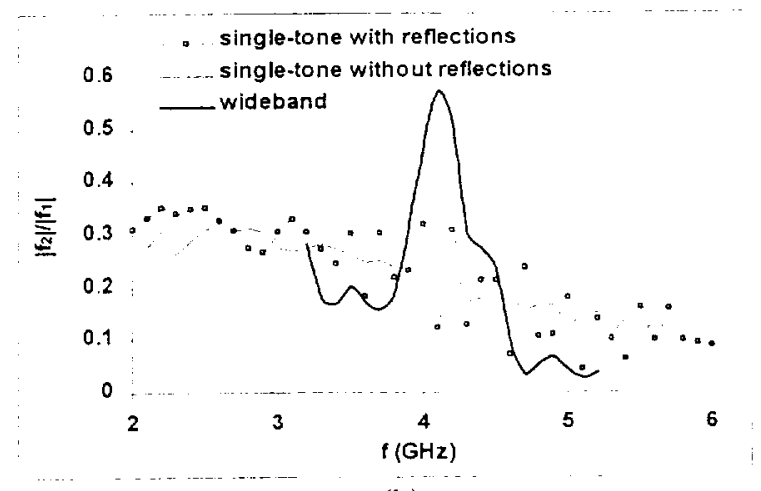

(b)

Figure 4 (a) Gain and (b) relative strength of second harmonic with and without reflections as a function of frequency

\section{Differences between broadband and single-tone} excitations

As an alternative to nonlinear, black box, TWT models using swept-amplitude data, box models based on swept-frequency, swept-amplitude data ${ }^{6}$ exist as well. Although this method accounts for the frequency dependent dispersion of the TWT, it makes the assumption that wideband TWT operation can be described by superimposing the effect of several singletone excitations. In addition, there is no inclusion of the effect of frequency dependent reflections. These assumptions were investigated by comparing the TWT characteristics when excited by single frequency tones versus wideband root raised cosine (RRC) shaped symbols, as would be used to represent realistic digital symbols to prevent intersymbol interference ${ }^{9}$.

A roll off factor, $\alpha$, of 0.3 was chosen for the RRC input, and the symbol width $\mathrm{T}$ was determined for a given bandwidth BW as

$T=\frac{1}{B W}(1+\alpha)$ seconds.

Several symbols were transmitted spaced $8 \mathrm{~T}$ seconds apart, allowing the signal to be repeatedly reflected so it 
travels the feedback cycle of the TWT several times. Including $8 \mathrm{~T}$ seconds for each symbol captures the majority of the energy within the chosen bandwidth. The average input power was kept at $5 \mathrm{~dB}$ IBO. The RF excitation was turned on at a point in time after the beam turn-on transient hits the output coupler. This is illustrated in Figure 5 where the input and output timedependent RRC symbols are shown for a four $\mathrm{GHz}$ bandwidth excitation, centered at $4.2 \mathrm{GHz}$. Strictly, these time-dependent signals represent the square root of the input/output power.

The gain and second harmonic strength of the last output symbol are compared to data using single-tone excitation with and without reflections in Figure 4 . The overall behaviors are very different for the single-tone and wideband excitations. The plots show that using single-tone excitation data without reflections would incorrectly predict gain by as much as about one $\mathrm{dB}$.

The large strength of the second harmonic at $8.4 \mathrm{GHz}$ for the wideband signal is due to the signal repeatedly being reflected, and repeatedly amplified in the forward direction. For these simulations we found that the second harmonic reached an equilibrium strength after the fifth RRC symbol out.

It should be mentioned that in an experimental TWT, losses will be present in the interaction circuit that will act to attenuate the second harmonic signal by a factor of about $\sqrt{2}$ compared to the signal at the fundamental frequency because of the scaling nature of conductor resistivity with frequency ${ }^{10}$. Also, the effects of the second harmonic can be mitigated in practice by inputting a signal at the second harmonic frequency, but with phase 180 degrees out of phase with the second harmonic signal generated by the nonlinearity of the TWT. This has the effect of canceling the unwanted second harmonic.

Neglecting losses will also increase the chance of backward wave oscillations (BWO) resulting from interaction of the $n=-1$ space harmonic with the beam. To ensure there was not a backward oscillation present, the time-dependent output signals and their respective frequency spectrums were monitored for all simulations. For this helical circuit, the voltage line intersects the $n=-1$ spatial harmonic at about $18 \mathrm{GHz}$ which is beyond the coupler passband. Thus, if there was a BWO because of the interaction with this $n=-1$ harmonic, it would manifest itself in the output as an intermodulation product. Operation at $4.2 \mathrm{GHz}$ would result in a third order intermodulation product at 18 $2 * 4.2=9.6 \mathrm{GHz}$. There was no evidence of a strong signal at this frequency.

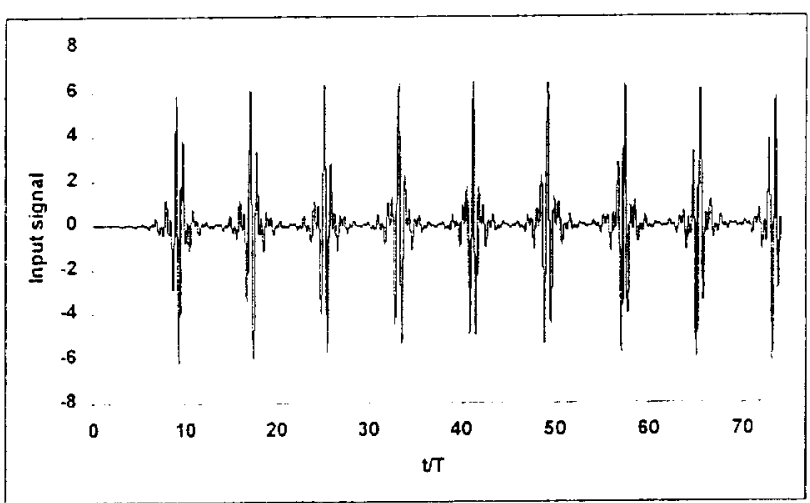

(a)

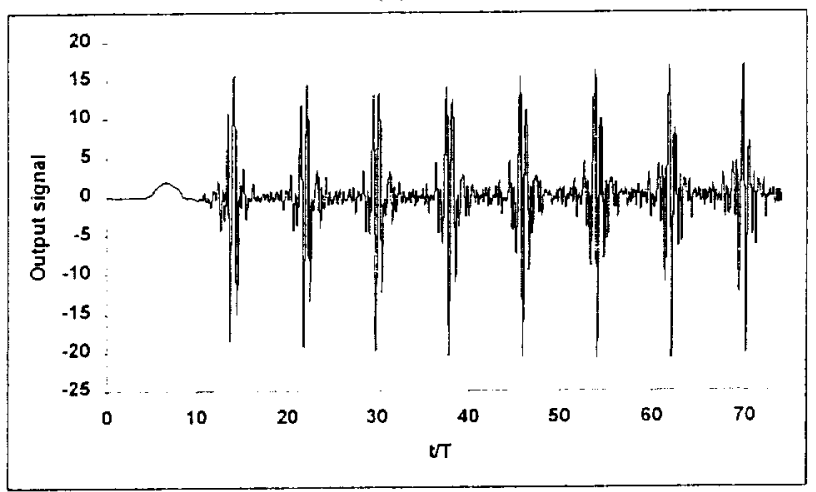

(b)

Figure 5 (a) Input and (b) output signals using several wideband root raised cosine shaped symbols $(\mathrm{BW}=4 \mathrm{GHz}, 5 \mathrm{~dB}$ IBO)

\section{Dependence of gain and phase ripples with drive power}

It is well known that the gain and phase ripples caused by the TWT vary with input drive power. It was shown in "that this ripple had a significant effect on bit error rate (BER) performance. However, gain and phase ripples are not accounted for in the SPW envelope model. In this section, the gain and phase ripples are calculated as a function of drive power using the MAFIA model by simulating two $\mathrm{GHz}$ bandwidth, RRC symbol trains similar to that shown in Figure 5 with various input powers. Within this bandwidth, the return loss is better than $-20 \mathrm{~dB}$ across the bandwidth, and there are no second harmonics within the operating band. Thus, it represents a more realistic scenario for communications applications. The normalized input spectrum of a single symbol, and the normalized output spectrums for the last symbols in each train are shown in Figure 6. A power hole (as much as about one $d B$ at 4.2 $\mathrm{GHz}$ ) exists in the operating band, increasing with input drive due to the second harmonic strength, which also increases with drive power reaching about $60 \%$ of the fundamental near saturation. The gain and phase ripples, shown in Figure 7, were calculated as the 
maximum deviation of the gain and phase, respectively, for the RRC output symbols. The largest values correspond to the largest IBO and decrease, in general, with increasing drive power. Comparing the gain ripple for the 5.1 IBO data in Figure 7 (a) to that in Figure 4

(a) for single tone excitations further demonstrates the differences in the TWT output as a function of excitation.

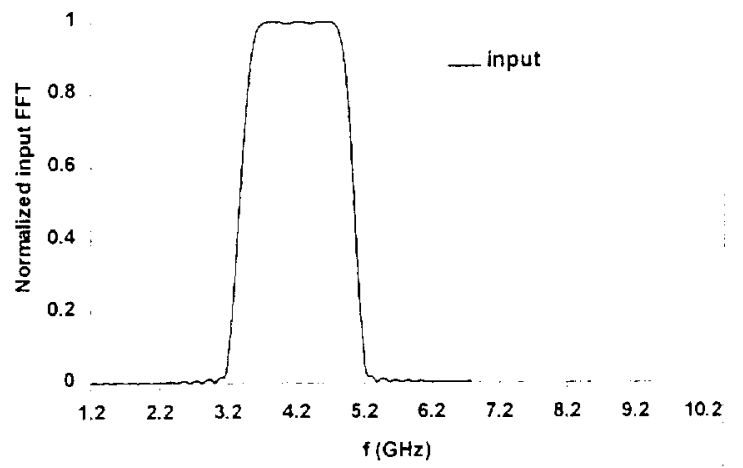

(a)

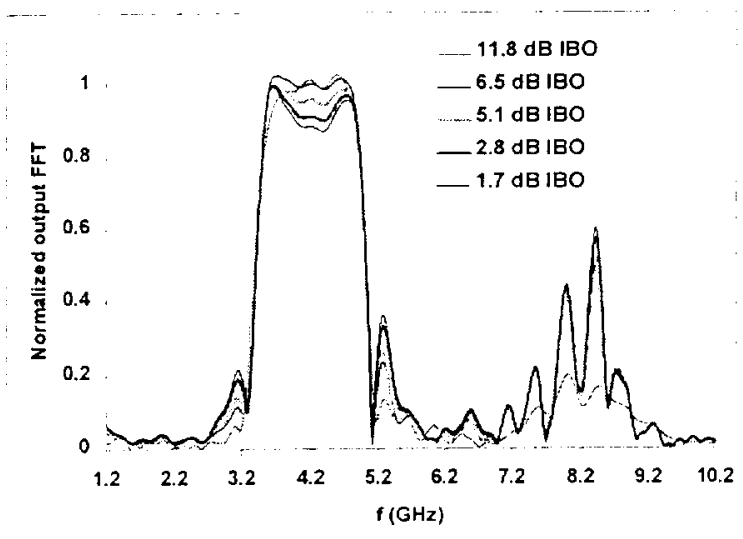

(b)

Figure 6 Simulated normalized (a) input and (b) output spectrums using wideband excitation at various levels of input backoff $(\mathrm{BW}=2 \mathrm{GHz})$

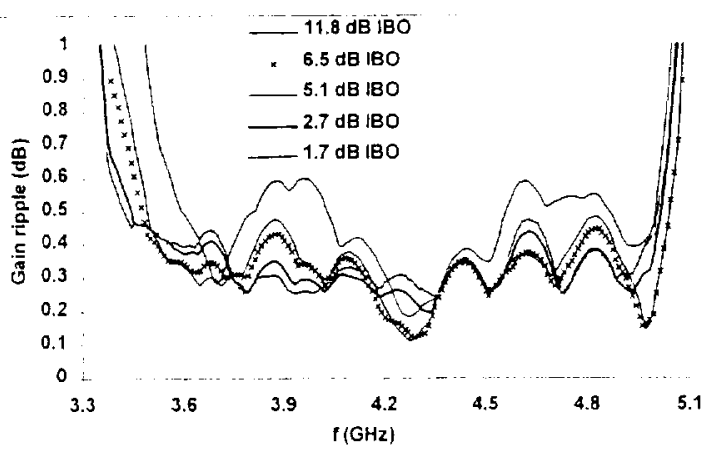

(a)

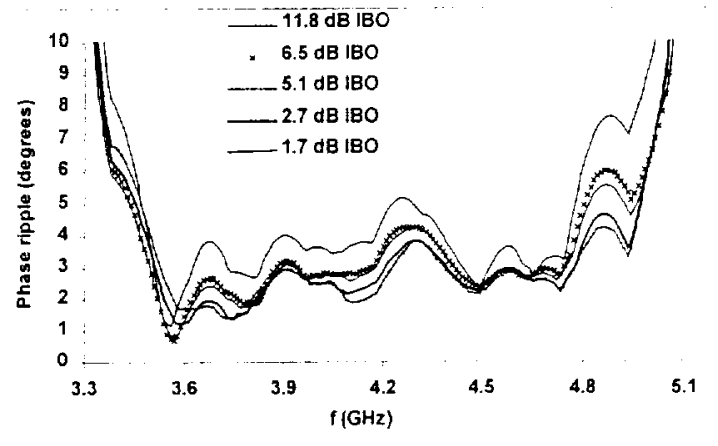

(b)

Figure 7 (a) Gain and (b) phase ripples using wideband excitation at various levels of input backoff $(\mathrm{BW}=2 \mathrm{GHz})$

\section{Dependence of output spectrum with operational signal}

To further demonstrate the idea that direct excitation with the operational signal provides superior simulated results, we compare the frequency spectrums of various modulation schemes. The bandwidth was kept constant at two $\mathrm{GHz}$ and the symbols were shaped using a RRC filter with $\alpha=0.3$, and a $T$ second spacing between symbols. Three cases are compared: 1) binary phase shift keying (BPSK), 2) 16-ary quadrature amplitude modulation (16-QAM), and 3) 64-QAM.

Figure 8 shows the output spectrum for each excitation, which consists of several tens of data symbols. The output spectrum was calculated for the entire output signal, as opposed to Figure 6 , which was calculated for a single symbol. The peak-to average power ratio $\mathrm{P}_{\mathrm{p}} / \mathrm{P}_{\mathrm{avg}}$ and IBO are specified in the figure caption. The differences in the output spectrums indicate the importance of considering the operational signal. 


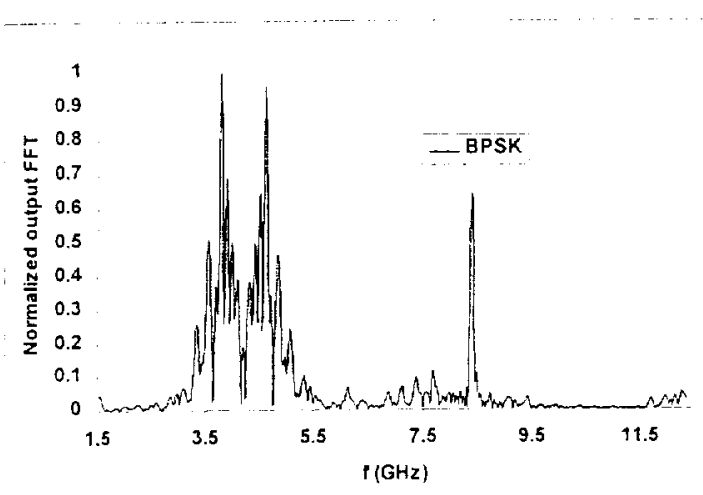

(a)

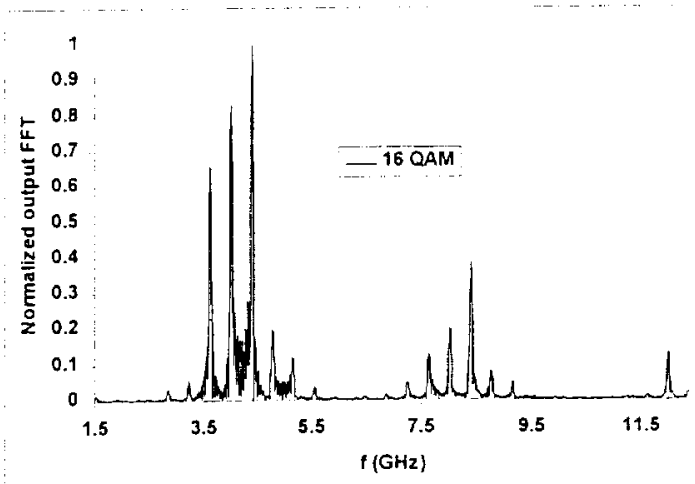

(b)

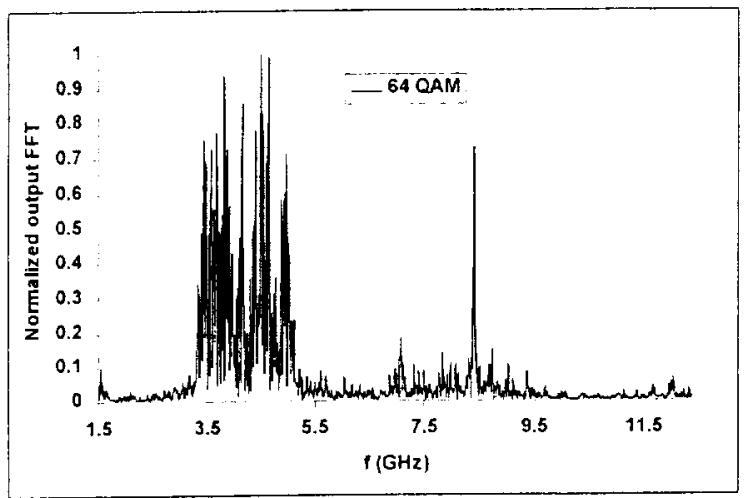

(c)

Figure 8 Normalized output spectrum for (a) BPSK (5.5 IBO, $P_{p} / P_{\text {avg }}=6.8 \mathrm{~dB}$ ), (b) 16-QAM (6.5 IBO, $\left.P_{p} / P_{\text {avg }}=6.5 \mathrm{~dB}\right)$, and (c) 64-QAM (7.5 IBO, $P_{p} / P_{\text {avg }}$ $=9 \mathrm{~dB})(\mathrm{BW}=2 \mathrm{GHz})$

\section{MAFIA AND SPW COMPARISONS}

Comparison are made using direct excitation of the MAFIA model and SPW simulations to quantitavely determine the effects of making the assumptions outlined in Section III on digital signal performance. The TWT box model used in the SPW simulations uses the MAFIA generated AM/AM and AM/PM data shown in Figure 2 in a lookup table format, and makes the assumptions listed in Section III.

The eye diagram is commonly used to qualitatively measure the degree of distortion due to ISI. Figure 9 shows eye diagrams generated from SPW and MAFIA output for a two $\mathrm{GHz}$ bandwidth, BPSK signal at a 5.5 $\mathrm{dB} I B O$. The absolute differences in upper bound degradation, which is proportional to the opening of the eye diagram ${ }^{6}$, between the SPW and MAFIA simulations as a function of signal bandwidth and IBO are shown in Figure 10. The discrepancies become worse with increased bandwidth (as larger reflections are present), and increased drive powers (as the power hole increases). Still, the considerable integrity of the BPSK output signal when subjected to high TWT distortion, shows that BPSK is not sensitive to ISI

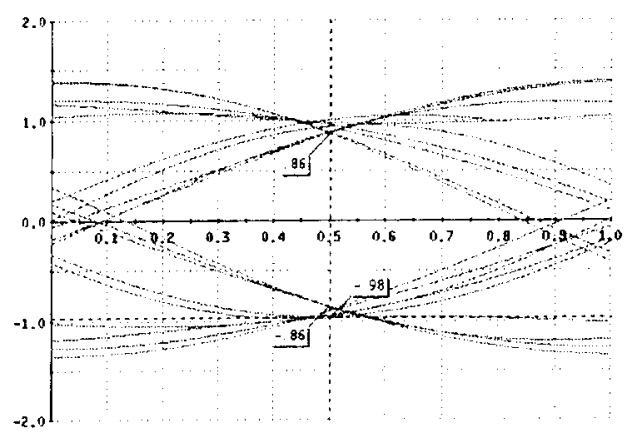

(a)

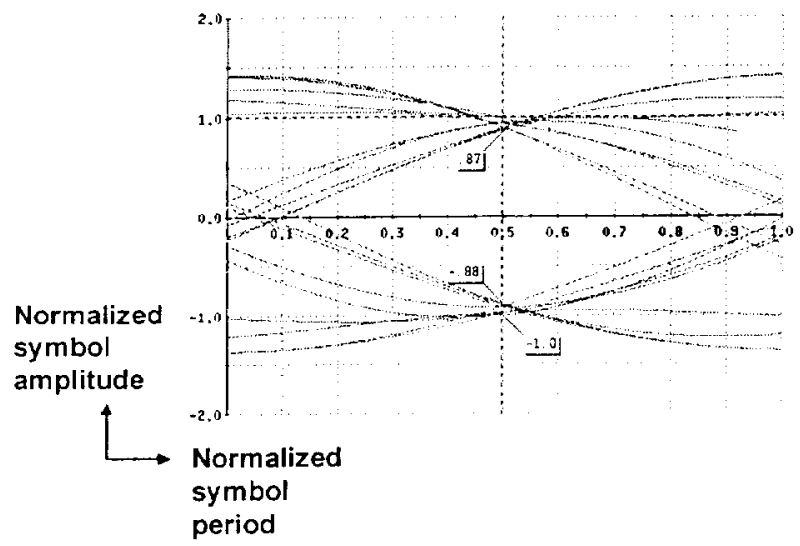

(b)

Figure 9 BPSK eye diagrams $(\mathrm{BW}=2 \mathrm{GHz}, 5.5 \mathrm{~dB}$ IBO) (a) SPW (b) MAFIA 


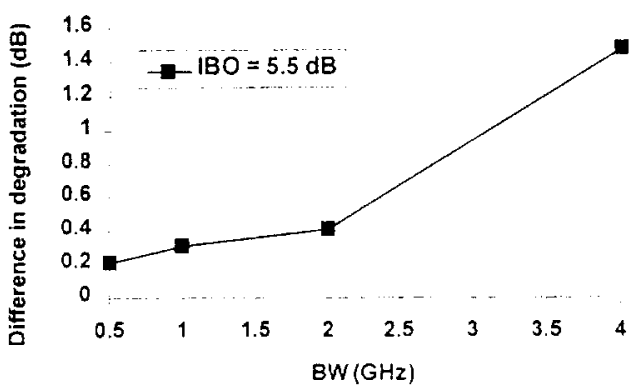

(a)

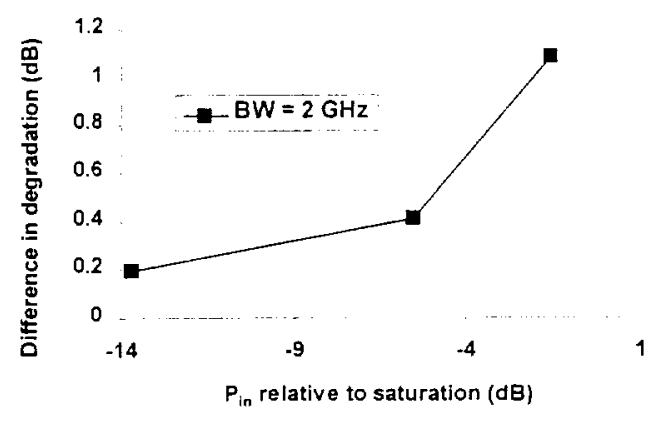

(b)

Figure 10 Difference in upper bound degradation using BPSK (a) $5.5 \mathrm{~dB} I \mathrm{BO}$, (b) $\mathrm{BW}=2 \mathrm{GHz}$

Constellation diagrams showing the output signal amplitude and phase for a 16-QAM signal are compared in Figure 11 (data generated for second and fourth quadrants to conserve computational time). SPW results were identical for two and four $\mathrm{GHz}$ BWs since frequency dependence is neglected. The upper bound degradation was calculated from the corresponding eye diagrams shown in Figure 12 for the in-phase (I) channel of the signal using the worst case eye opening. The MAFIA constellation diagram shows spreading due to ISI. This is manifested as an eye closing in the eye diagrams of Figure 12. Compared to SPW simulations, the MAFIA two and four $\mathrm{GHz}$ cases resulted in larger degradations by 1.6 and $3.6 \mathrm{~dB}$, respectively, implying a heightened degree of sensitivity to ISI as compared to BPSK.

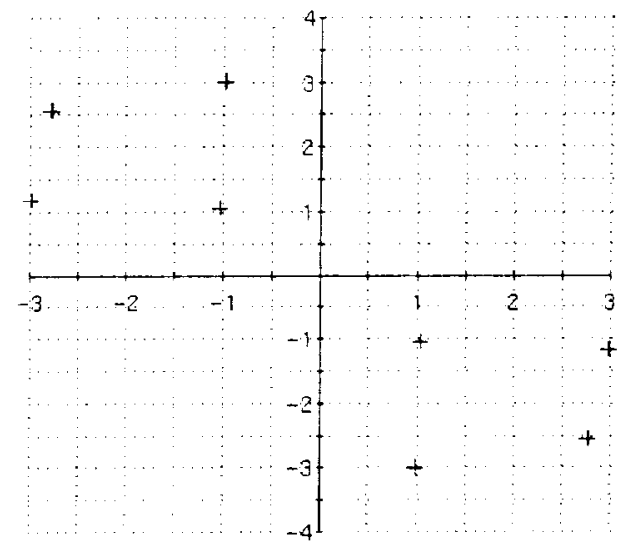

(a)

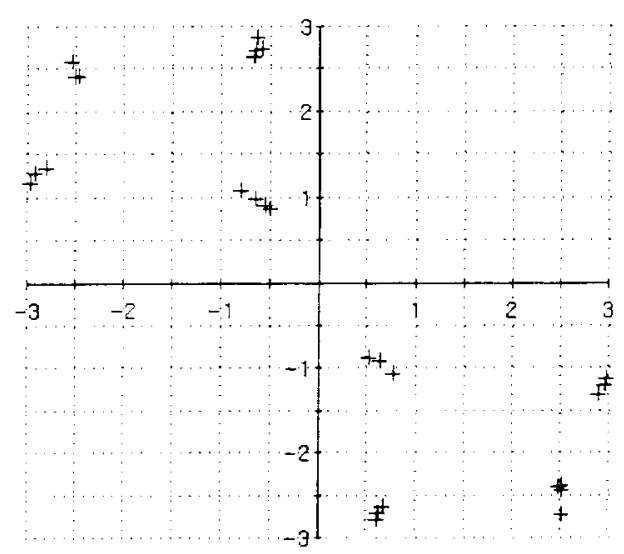

(b)

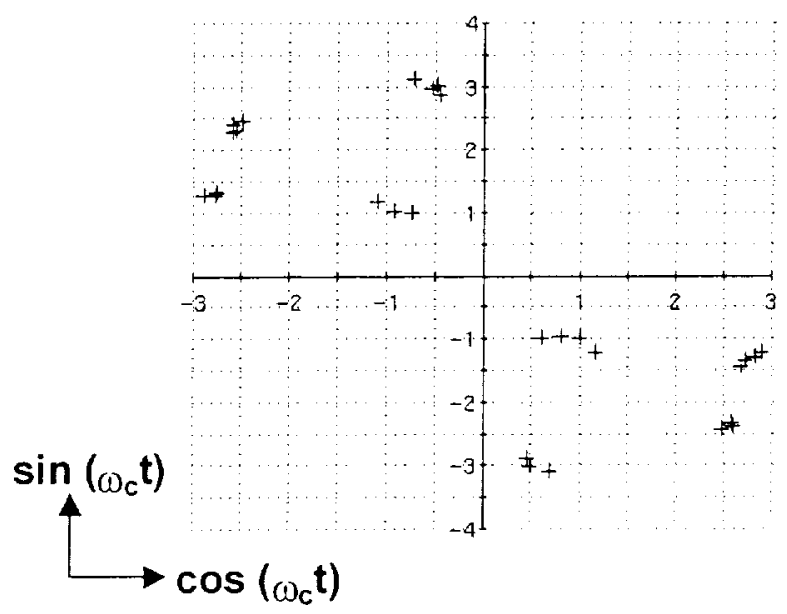

(c)

Figure 11 16-QAM constellation diagrams $(6.5 \mathrm{~dB}$ IBO) (a) SPW ( 2 and $4 \mathrm{GHz}$ BWs), (b) MAFIA (BW $=2 \mathrm{GHz})$, and $(\mathrm{c}) \mathrm{MAFIA}(\mathrm{BW}=4 \mathrm{GHz})$ 


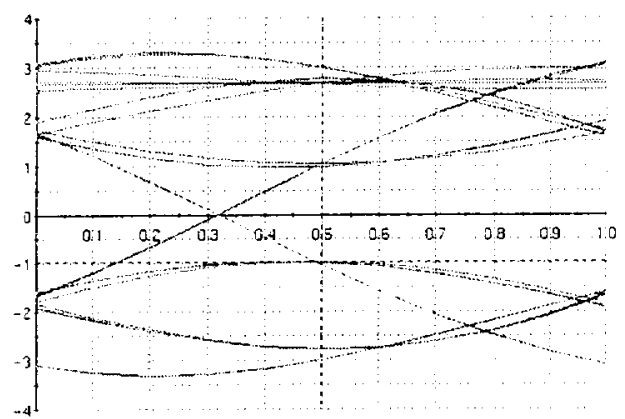

(a)

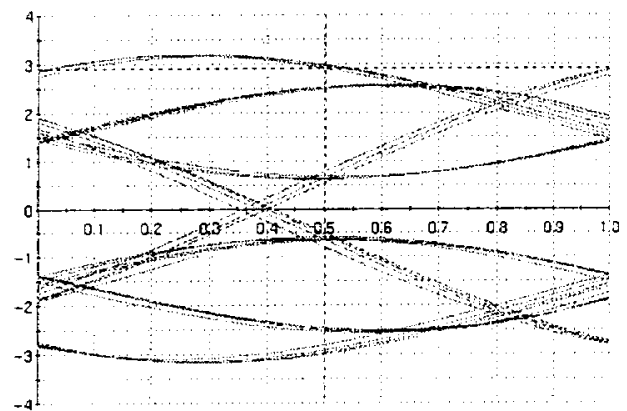

(b)

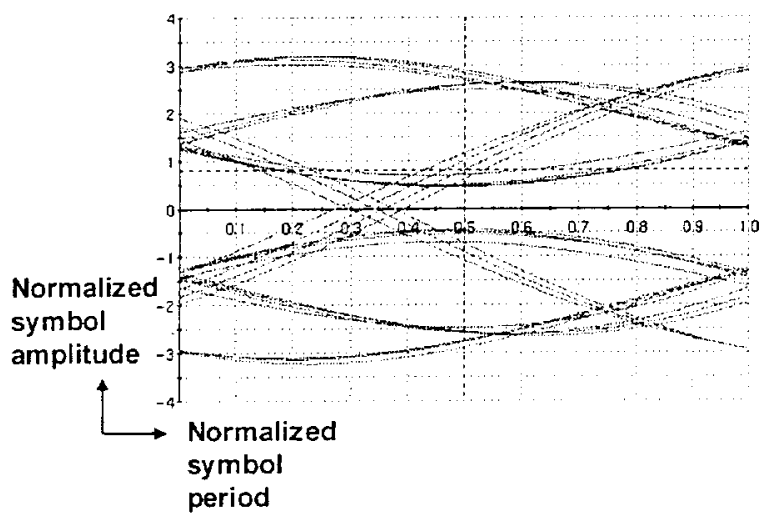

(c)

Figure 12 16-QAM eye diagrams for in-phase (I) channel (6.5 dB IBO) (a) SPW (2 and $4 \mathrm{GHz}$ BWs), (b) MAFIA (BW $=2 \mathrm{GHz}$ ), and (c) MAFIA (BW $=4$ $\mathrm{GHz}$ )

Lastly, 64-QAM was used to excite the MAFIA and SPW models with various bandwidths including one, two and four GHz. The ideal, SPW and MAFIA constellation diagrams are shown for the second and fourth quadrants in Figure 13. Again, the SPW output is constant for each case since the TWT model has no frequency dependence. The corresponding eye diagrams are shown in Figure 14. As evidenced by the plots, the distortion is severe for both the SPW and MAFIA simulations. This would indicate a significant portion of the distortion is caused by the nonlinearity of the TWT (AM/AM and AM/PM conversion), and operating the TWT at a larger input backoff could reduce this. The discrepancy between the SPW and MAFIA simulations shows the added distortion from ISI, which increases with bandwidth. Because of the severe distortion the upper bound degradation is difficult to calculate. The sensitivity of 64-QAM to both TWTA nonlinearity and ISI is readily apparent from these simulations. 
(a)

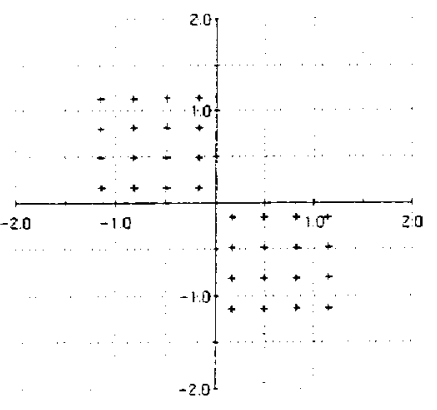

(b)

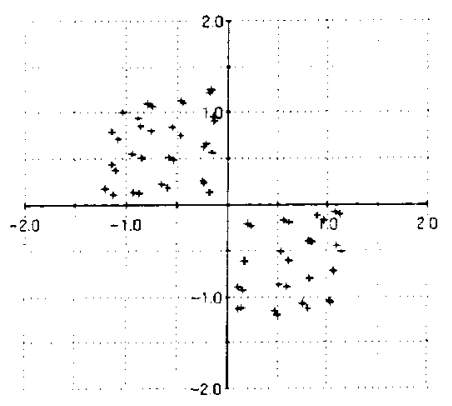

(c)

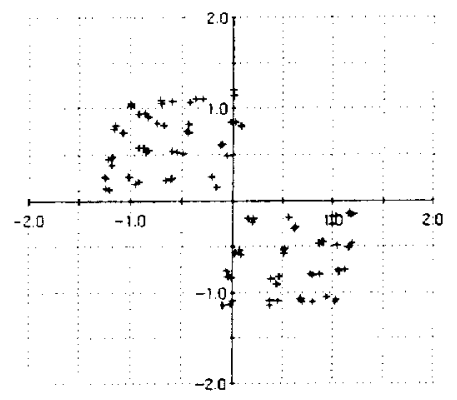

(d)

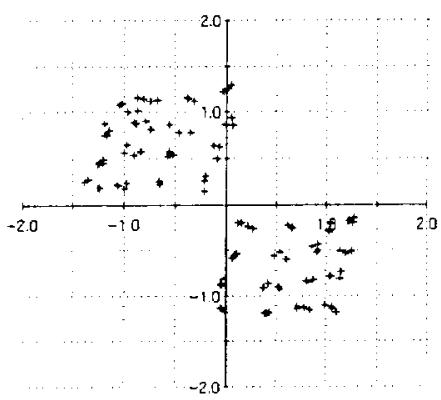

(e)

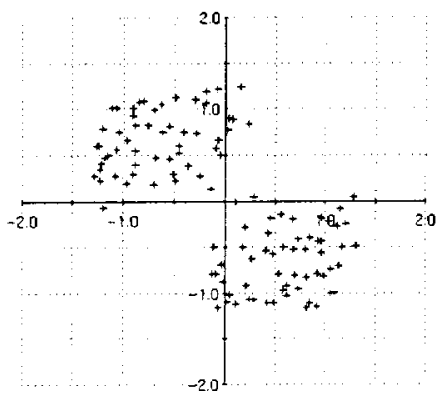

Figure 13 64-QAM constellation diagrams $(7.5 \mathrm{~dB}$ IBO) (a) Ideal output (b) SPW output (1, 2 and 4 GHz BW), (c) MAFIA output, (1 GHz BW), (d)

MAFIA output, (2 GHz BW), (e) MAFIA output, (4 $\mathrm{GHz}$ BW) (a)

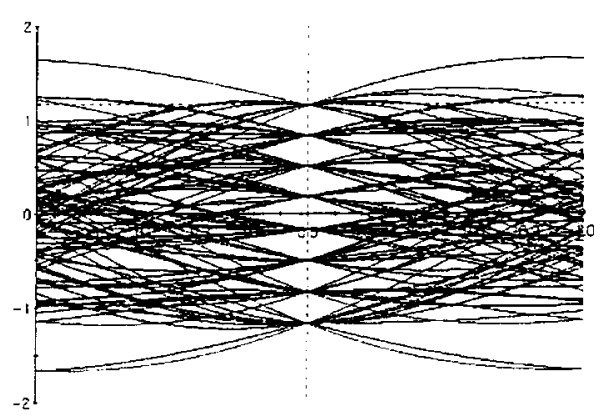

(b)

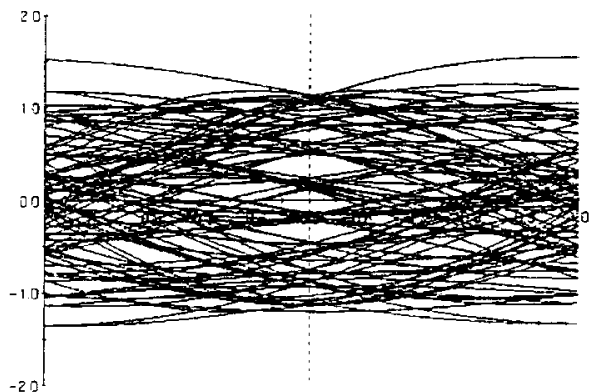

(c)

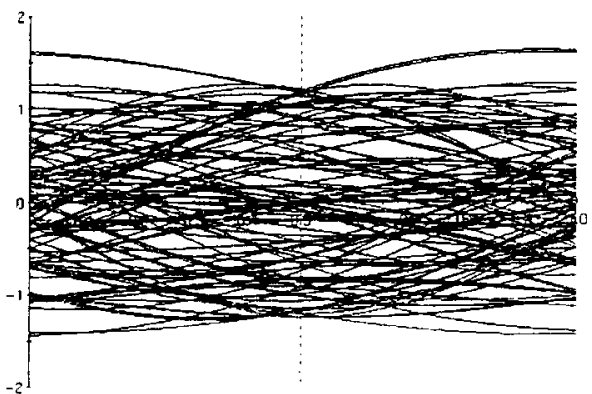

(d)

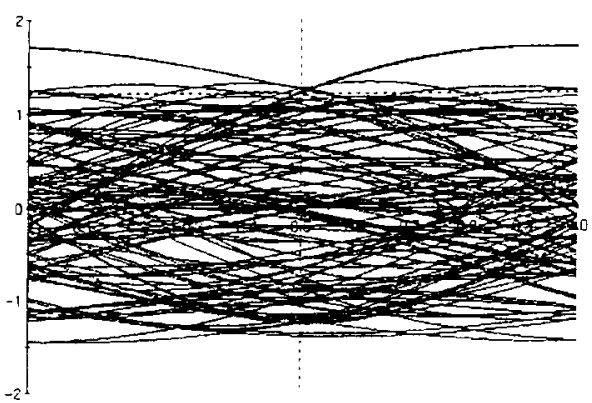

(e)

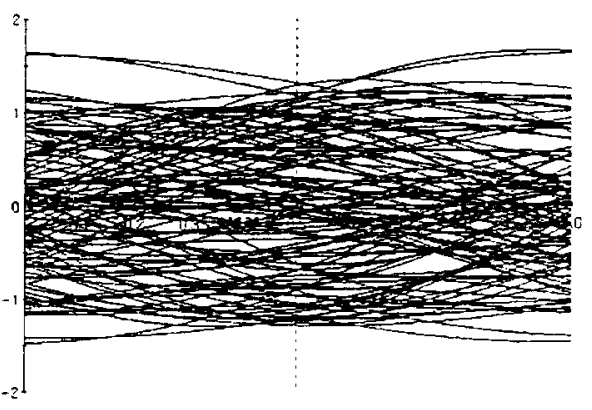

Figure 14 64-QAM eye diagrams (7.5 dB IBO) (a) Ideal output (b) SPW output (1, 2 and $4 \mathrm{GHz} B W)$, (c) MAFIA output, (1 GHz BW), (d) MAFIA output, (2 GHz BW), (e) MAFIA output, (4 GHz BW) 


\section{CONCLUSIONS}

A 3D MAFIA time-dependent TWT interaction model was presented, and used to investigate assumptions made in TWT black box models used in communication system level simulations. In addition, direct digital data were used as input into the MAFIA model and signal degradation due to the TWT was compared with SPW simulations. Results show significant differences in predicted degradation between SPW and MAFIA simulations, giving an indication of the effect of ISI on performance. This demonstrates the significance of the approximations made in the SPW model regarding the TWT characteristics on digital signal performance, and the superior predicative capability of using the timedependent MAFIA TWT model. These 3D MAFIA simulations are presently computationally intensive (several hours); thus, more accurate, system level, black-box models with memory are being developed using MAFIA time-dependent output to determine the model parameters.

Acknowledgement - The authors would like to thank Gary Groshart (Northrop Grumman), John Booske and Mark Converse (University of Wisconsin, Madison) for providing the experimental data, and Dave Aster (Asgard Microwave) for his assistance in the coupler design.

\section{REFERENCES}

\footnotetext{
${ }^{1} \mathrm{SPW}^{\mathrm{TM}}$, Signal Processing Worksystem. Comdisco Systems, Inc. 919 Hillsdale Blvd., Foster City, CA 94404.

${ }^{2}$ D. M. MacGregor, Two-dimensional nonlinear multisignal helix traveling-wave tube amplifier computer program, Volume 1: User Manual, Electrocon International, Inc., Ann Arbor, Michigan, April 1993.

${ }^{3}$ C L L. Kory, Three-dimensional simulations of PPM Focused Helical Traveling-wave tubes, Doctor of Engineering Dissertation, Cleveland State University, Cleveland, Ohio, August 2000.

${ }^{4} \mathrm{~T}$. Weiland, On the numerical solution of Maxwell's equations and applications in the field of accelerator physics, Part. Accel., Vol. 15, pp. 245-292, 1984.

${ }^{5} \mathrm{~T}$. Weiland, On the unique numerical solution of Maxwellian eigenvalue problems in three dimensions, Part. Accel., Vol. 17, pp. 227-242, 1985.

${ }^{6}$ M. C. Jeruchim, P. Balaban, and K. S. Shanmugan, Simulation of Communication Systems. Modeling, Methodology, and Techniques, $2^{\text {nd }}$ Edition, Kluwer Academic/Plenum Publishers, New York (2000).
}

${ }^{7}$ C. J. Clark, G. Chrisikos, M. S. Muha, A. A. Moulthrop and C. P. Silva, Time-domain envelope measurement technique with application to wideband power amplifier modeling, IEEE Trans. Microwave Theory and Techniques, Vol. 46, No. 12, Dec. 1998. ${ }^{8}$ J. W. Hansen, G. A. Lange, A. S. Rostad and R. L. Woods, Hughes Aircraft Company Electron Dynamics Division Applications Note System Aspect of Communications TWTAs, August 1982.

9 Andy Bateman, Digital Communications-Design for the Real World, Addison-Wesley, Edinburgh Gate, UK, 1999.

${ }^{10}$ S. Ramo, J. R. Whinnery, and T. Van Duzer, Fields and Waves in Communication Electronics, Third edition, John-Wiley and Sons, New York, NY, 1994.

${ }^{11} \mathrm{~K}$. Shalkhauser and G. Fujikawa, Bit-error-rate testing of high power $30 \mathrm{GHz}$ traveling wave tubes for ground terminal applications, NASA Technical Report 2635 , October 1986 